\title{
The Proteasome Regulates the Interaction Between Cx43 and ZO-1
}

\author{
Henrique Girao and Paulo Pereira* \\ Centre of Ophthalmology, Biomedical Institute for Research in Light and Image (IBILI), \\ Faculty of Medicine, University of Coimbra, 3000-354 Coimbra, Portugal
}

\begin{abstract}
Gap junction (GJ) intercellular communication (GJIC) is vital to ensure proper cell and tissue function. GJ are multimeric structures composed of proteins called connexins. Modifications on stability or subcellular distribution of connexins have a direct impact on the extent of GIIC. In this study we have investigated the role of the proteasome in regulation of connexin 43 (Cx43) internalization. Although the participation of both the proteasome and lysosome has long been suggested in Cx43 degradation, the molecular mechanisms whereby proteasome contributes to regulate $\mathrm{Cx} 43$ internalization and intercellular communication are still unclear. The results presented in this study envision a new mechanism whereby proteasome regulates GJIC by modulating interaction between CX43 and ZO-1. Immunoprecipitation experiments, in the presence of proteasome inhibitors, together with immunofluorescence data indicate that the proteasome regulates interaction between $\mathrm{Cx} 43$ and ZO-1. Overexpression of the PDZ2 domain of ZO-1 and the expression of $\mathrm{CX}-43$ fused in frame with a V5/HIS tag, suggest that interaction between the two proteins occurs through the PDZ2 domain of ZO-1 and the C-terminus of Cx43. When interaction between Cx43 and ZO-1 is reduced, as in the presence of proteasome inhibitors, $\mathrm{C} \times 43$ accumulates, forming large GJ plaques at plasma membrane. Data presented in this article suggest a new pathway whereby alterations in proteasome activity may impact on GIIC as well as on non-junctional communication with extracellular environment, contributing to cell and tissue dysfunction. J. Cell. Biochem. 102: 719-728, 2007. (c) 2007 Wiley-Liss, Inc.
\end{abstract}

Key words: connexin43; gap junction; proteasome; ubiquitin; ZO-1; endocytosis

Gap junctions (GJ) are intercellular channels that permit the passage of small molecules such as metabolites, ions, and second messengers [Kumar and Gilula, 1996]. Exchange of low molecular weight substances through gap junctions is an important mechanism for cells to regulate homeostasis, proliferation, differentiation and to ensure homogeneous tissue function [Simon and Goodenough, 1998; Matemba et al., 2006]. Gap junctions consist of two hemichannels, called connexons, that are located in the plasma membrane of two adjacent cells. Each connexon is composed of six subunits of a protein called connexin $(\mathrm{Cx})$.

The extent of gap junction intercellular communication is a direct result of the number

*Correspondence to: Paulo Pereira, Azinhaga Sta Comba, 3000-354 Coimbra, Portugal. E-mail: ppereira@ibili.uc.pt

Received 15 September 2006; Accepted 8 February 2007

DOI 10.1002/jcb.21351

(c) 2007 Wiley-Liss, Inc. and functionality of these connexin-based pores. In addition to regulation by transcriptional control, other rapid mechanisms are also involved in regulation of gap junction intercellular communication. These mechanisms include altered subcellular localization and a variety of post-transcriptional modifications such as phosphorylation and rapid degradation of connexins. Both the proteasome [Laing and Beyer, 1995; Beardslee et al., 2000; Musil et al., 2000; Girao and Pereira, 2003; Fernandes et al., 2004 Laing et al., 2005] and lysosome [Laing et al., 1997; Musil et al., 2000] have been implicated in degradation of $\mathrm{Cx} 43$. Whereas degradation of $\mathrm{Cx} 43$ by the lysosome has long been established, the role of the proteasome in $\mathrm{Cx} 43$ turnover is still poorly understood. Initial studies suggested that the proteasome could be directly involved in connexin degradation [Laing and Beyer, 1995]. However, more recently, it has been hypothesized that proteasome acts by regulating the stability of $\mathrm{Cx} 43$ at the plasma membrane, most likely by degrading a connexin-interacting 
protein [Musil et al., 2000; Girao and Pereira, 2003]. Indeed, it has been demonstrated that proteasome inhibition is associated with an increase in gap junctional plaque assembly and intercellular dye transfer, suggesting that this proteolytic complex is somehow involved in internalization of $\mathrm{Cx} 43$.

$\mathrm{Cx} 43$ is known to interact with several proteins, including cytoskeletal proteins and anchoring proteins, such as E-cadherin [Fujimoto et al., 1997], caveolin-1 [Schubert et al., 2002], and ZO-1 [Giepmans and Moolenaar, 1998; Toyofuku et al., 1998; Wu et al., 2003] that may regulate channel assembly and/or the stability of $\mathrm{Cx} 43$ at the plasma membrane. The interaction of $\mathrm{Cx} 43$ with zonula occludens (ZO1 ) has been studied in great detail and several studies have focused on the mechanisms and functional implications of such interaction. ZO1 is a member of the membrane-associated guanylate kinase (MAGUK) family, that is, important in regulation of signal transduction, protein targeting and control of cell polarity [Gonzalez-Mariscal et al., 2000; Harhaj and Antonetti, 2004]. Immunoprecipitation and yeast-two-hybrid experiments have demonstrated that interaction between $\mathrm{ZO}-1$ and $\mathrm{Cx} 43$ occurs through the last five aminoacids (DDLEI) of the C-terminus (CT) of $\mathrm{Cx} 43$ and the second PDZ domain (PDZ2) of ZO-1 [Giepmans and Moolenaar, 1998; Giepmans et al., 2001; Singh and Lampe, 2003; Jin et al., 2004; Sorgen et al., 2004]. Moreover, studies using mutant connexin peptides that lack the CT binding domain or in which the CT is blocked, revealed that formation of GJ can occur without interaction of $\mathrm{Cx} 43$ with ZO-1 [Jordan et al., 1999; Bukauskas et al., 2000; Toyofuku et al., 2001b]. However, the GJ formed under such conditions are abnormally large and some of the channels are inactive, suggesting that ZO-1 is somehow involved in regulation of GJ function [Hunter et al., 2003; 2005; Jin et al., 2004]. The implications and the physiological role of the interaction between $\mathrm{Cx} 43$ and $\mathrm{ZO}-1$ remain controversial. Early studies hypothesized that ZO-1 could act as an anchor stabilizing GJ at plasma membrane, most likely through cytoskeletal anchoring [Toyofuku et al., 1998, 2001b]. More recently, it was demonstrated that interaction between $\mathrm{Cx} 43$ and $\mathrm{ZO}-1$ is a dynamic process that is involved in the regulation of Cx43 endocytosis [Barker et al., 2002; Duffy et al., 2004; Segretain et al., 2004].
However, the precise role of $\mathrm{ZO}-1$ in $\mathrm{Cx} 43$ endocytosis is still controversial. Indeed, studies carried out in Sertoli cells and cardiac myocytes showed that $\mathrm{Cx} 43$ interacts more strongly with ZO-1 during endocytosis of GJ [Barker et al., 2002; Segretain et al., 2004]. Conversely, data obtained by Duffy suggested that internalization of GJs, induced by intracellular acidification, is facilitated by dissociation of Cx43 from ZO-1 [Duffy et al., 2004].

More recently it was demonstrated that interaction between $\mathrm{Cx} 43$ and $\mathrm{ZO}-1$ might act as a regulator of the size of GJ, by controlling the rate of channel accretion at plaque periphery [Hunter et al., 2005]. Indeed, when interaction between $\mathrm{Cx} 43$ and $\mathrm{ZO}-1$ is reduced, such as by the attachment of a tag to the C-terminus of $\mathrm{Cx} 43$ or by the use of a peptide inhibitor that contains the $\mathrm{Cx} 43 \mathrm{PDZ}$-binding domain, there is a reduction of ZO-1 peripherally associated with GJ and a significant increase in plaque size.

However, the regulation of the interaction between ZO-1 and $\mathrm{Cx} 43$ still remains to be elucidated. In this study we provide evidence that the proteasome plays an important role in regulating interaction between $\mathrm{Cx} 43$ and $\mathrm{ZO}-1$. Indeed, the data presented in this study show that proteasome inhibition results in a decreased interaction between $\mathrm{Cx} 43$ and ZO-1. Moreover, the presence of a tag in the C-terminus of $\mathrm{Cx} 43$, that prevents interaction with $\mathrm{ZO}-1$, reverts the MG132-induced accumulation of large $\mathrm{Cx}-43$ plaques at the plasma membrane. These data suggest that efficient interaction between $\mathrm{Cx} 43$ and $\mathrm{ZO}-1$ is regulated by the proteasome, presumably by involving degradation of a putative $\mathrm{Cx} 43$ binding protein.

\section{MATERIALS AND METHODS}

\section{Antibodies}

The rabbit anti-Cx43 polyclonal antibody, obtained from Zymed was raised against a peptide corresponding to a segment of the third cytoplasmic domain (C-terminal portion) of rat Cx43 (Cat No 71-0700). The mouse anti-Cx43 monoclonal antibody obtained from Zymed was raised against a cytoplasmic sequence located near the C-terminus of rat $\mathrm{Cx} 43$ (Cat No 13-8300). The mouse anti-Cx43 monoclonal antibody obtained from BD Tranduction Laboratories was raised against a peptide sequence that represents amino acid residues 252-270 of 
rat $\mathrm{Cx} 43$. Polyclonal antibodies against ZO-1 were obtained from Zymed.

\section{Culture and Treatment of Cells}

NRK cells or HEK293 cells were cultured in Dulbecco's Modified of Eagle's Medium (DMEM) supplemented with $10 \%$ fetal bovine serum and penicillin/streptomycin. To determine the role of the proteasome on $\mathrm{Cx} 43$ interaction with $\mathrm{ZO}-1$, cells were incubated, for $2 \mathrm{~h}$, in the presence of the proteasome inhibitors MG132 $(40 \mu \mathrm{M})$ or Lactacystin $(10 \mu \mathrm{M})$.

\section{Cell Transfection}

Transient transfections of HEK 293 cells were performed by incubating the cells with LipofectAMINE reagent in low serum medium (Life Technologies), during $6 \mathrm{~h}$. Plasmids encoding full length $\mathrm{Cx} 43$ or HIS-V5-tagged $\mathrm{Cx} 43$ were cloned into the pcDNA3.1/V5/His vector (Invitrogene).

\section{Immunoprecipitation and Western Blotting}

NRK cells or HEK293 transfected cells were rinsed with $\mathrm{PBS}$ at $4{ }^{\circ} \mathrm{C}$, ressuspended in lysis buffer $(190 \mathrm{mM} \mathrm{NaCl}, 50 \mathrm{mM}$ Tris-HCl, $6 \mathrm{mM}$ EDTA, 1\% Triton X-100, pH 8.3) supplemented with protease inhibitor cocktail (Roche), $2 \mathrm{mM}$ PMSF, $10 \mathrm{mM}$ iodacetamide, $50 \mathrm{mM} \mathrm{NaF}, 500 \mu \mathrm{M}$ $\mathrm{NaVO}_{4}$, and incubated on ice during $30 \mathrm{~min}$. The samples were then centrifuged at $10,000 \mathrm{~g}$, during $10 \mathrm{~min}$, and the supernatants used for immunoprecipitation. Briefly, protein A was incubated with polyclonal antibodies directed against Cx43 (Zymed, Cat No 71-0700), for $1 \mathrm{~h}$, at $4{ }^{\circ} \mathrm{C}$ or nonimmune rabbit serum, followed by incubation with supernatants, for $3 \mathrm{~h}$ at $4^{\circ} \mathrm{C}$. The samples were then centrifuged and the protein A-sepharose sediments ressuspended in Laemmli buffer and denatured at $37^{\circ} \mathrm{C}$, for $30 \mathrm{~min}$.

For Western blot analysis of the immunoprecipitated proteins, samples were separated by SDS-PAGE, transferred to a PVDF membrane, and labeled with monoclonal antibodies to $\mathrm{Cx} 43$ (Zymed, Cat No 13-8300) or polyclonal antibodies to ZO-1.

\section{Immunofluorescence}

Cells grown on glass coverslips were fixed with $4 \%$ paraformaldehyde in phosphate buffered saline PBS. The samples were then washed with PBS, permeabilised with $0.2 \% \mathrm{v} / \mathrm{v}$ Triton X-100 in PBS, and blocked with goat serum (1:10) for 20 min prior to incubation with primary antibodies. Incubation with primary antibodies against $\mathrm{Cx} 43$ (Transduction Lab) and ZO-1 (Zymed) proceeded for $1 \mathrm{~h}$ at room temperature. The samples were then washed three times with PBS before incubation with the secondary antibody for $1 \mathrm{~h}$ at room temperature. The specimens were rinsed in PBS and mounted with Glycergel (Dako). All solutions were made up in $0.2 \% \mathrm{w} / \mathrm{v}$ BSA (Sigma) containing $0.02 \%$ sodium azide (Sigma) in PBS. For controls, primary antibodies were omitted. The images were collected by confocal microscopy using a Carl Zeiss LSM410 system and Bio-Rad MRC-600.

\section{RESULTS}

Previous studies showed that inhibition of Cx43-ZO-1 interaction results in a significant increase in the size of GJ plaques [Hunter et al., 2003; 2005; Jin et al., 2004]. On the other hand, it was shown that proteasome inhibition leads to the accumulation of $\mathrm{Cx} 43$ at the plasma membrane, with the formation of large plaques [Musil et al., 2000; Girao and Pereira, 2003; Fernandes et al., 2004]. This led us to hypothesize that an active proteasome is required to regulate the interaction of $\mathrm{Cx} 43$ with $\mathrm{ZO}-1$.

To investigate the role of the proteasome in Cx43-ZO-1 interaction, NRK cells, that endogenously express $\mathrm{Cx} 43$ and $\mathrm{ZO}-1$, were incubated either in the presence or absence of the proteasome inhibitor MG132 (or lactacystin), for $2 \mathrm{~h}$. To further evaluate the association of $\mathrm{Cx} 43$ and ZO-1, Cx43 or ZO-1 was selectively immunoprecipitated using polyclonal antibodies against each of these proteins, followed by Western blot and probing with antibodies

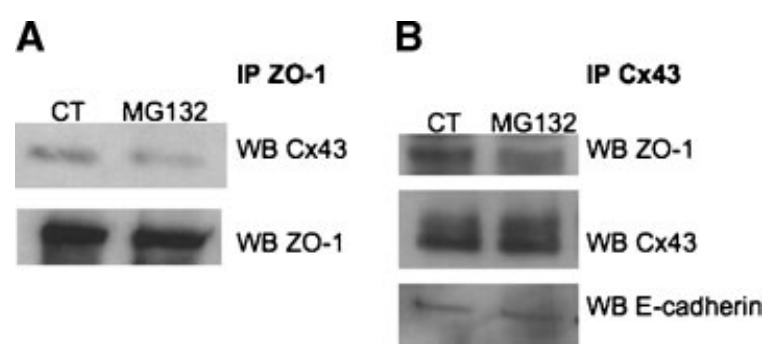

Fig. 1. Proteasome inhibitors reduce the interaction between Cx43 and ZO-1. Endogenous Cx43 from NRK cells was immunoprecipitated using polyclonal antibodies against ZO-1 (A) or Cx43 (B). Immunoprecipitated material was resolved by SDS-PAGE and immunoblotted with antibodies against $\mathrm{Cx} 43$ (A) or ZO-1 and E-cadherin (B). 
against ZO-1 or Cx43, respectively (Fig. 1). The results obtained clearly show that, in the cells incubated with MG132 (or lactacystin), the amount of $\mathrm{Cx} 43$ immunoprecipitated with anti-ZO-1 antibodies (Fig. 1A), and the amount of ZO-1 immunoprecipitated with anti-Cx43 antibodies (Fig. 1B), is lower than in control cells. These results demonstrate, for the first time, that proteasome inhibition leads to a decreased interaction between $\mathrm{Cx} 43$ and ZO-1.

To further investigate if the effect of proteasome inhibitors in binding of $\mathrm{Cx} 43$ to ZO- 1 is specific, cell lyzates were immunoprecipitated with anti-Cx43 antibodies and were subsequently Western blotted with antibodies against E-cadherin, which is known to interact with $\mathrm{Cx} 43$. The results presented in Figure 1B show that interaction between $\mathrm{Cx} 43$ and E-cadherin is not affected by proteasome inhibitors. This suggests that it is not likely that proteasome inhibitors are unspecifically disrupting interaction between $\mathrm{Cx} 43$ and its binding partners. To verify that the effect of MG132 on the amount of immunoprecipitated proteins is not due to variations in the initial amount of proteins, we reprobed the same membranes with the antibodies used in the immunoprecipitation assays. The results show that the amount of $\mathrm{Cx} 43$ and ZO-1 are not significantly altered following incubation with MG132. In the immunoprecipitated $\mathrm{Cx} 43$ there is an accumulation of slower migrating bands that correspond to the phosphorylated forms of the protein (Fig. 1B, middle panel). Additional controls were generated by probing the membranes, containing extracts before immunoprecipitation with Ponceau S or with antibodies against actin. In both cases the staining for the different samples was comparable, indicating equal sample loading (data not shown).

To further confirm the decrease of $\mathrm{Cx} 43-\mathrm{ZO}-1$ interaction induced by proteasome inhibitors, NRK cells, that endogenously express $\mathrm{Cx} 43$, were incubated either in the presence or absence of MG132 (or lactacystin), and then fixed and stained with monoclonal antibodies directed against $\mathrm{Cx} 43$ and polyclonal antibodies against ZO-1. Cells were subsequently imaged by immunofluorescence confocal microscopy. The results show that, in controls, the majority of $\mathrm{Cx} 43$ colocalizes with ZO-1 (Fig. $2 \mathrm{~A} \mathrm{a}-\mathrm{c}$ ) at the plasma membrane, while in cells incubated with MG132 it is possible to observe the formation of large punctate staining (Fig. $2 \mathrm{~A} \mathrm{~d}-\mathrm{f}$; see arrows) corresponding to GJ plaques that do not colocalize with ZO-1 (Fig. 2A f; see arrows). To confirm that this does not result from the fixation procedure, in addition to PFA, cells were also fixed with methanol/acetone (Fig. 2B). Taken together, these results suggest that efficient interaction between $\mathrm{Cx} 43$ and ZO-1 requires an active proteasome. Based on this observation and previous reports it is possible to speculate that proteasome inhibitors may prevent degradation of a putative $\mathrm{Cx} 43$ interacting protein, thus preventing an efficient association between $\mathrm{Cx} 43$ and $\mathrm{ZO}-1$.

To confirm that the binding of a protein to the C-terminus of $\mathrm{Cx} 43$ is sufficient to disrupt or weaken its interaction with $\mathrm{ZO}-1$, we added a tag to the C-terminus of $\mathrm{Cx} 43$. HEK239 cells, that do not endogenously express $\mathrm{Cx} 43$ [Toyofuku et al., 1998, 2001a,b], were transiently transfected with a plasmid containing the cDNA for the full length of $\mathrm{Cx} 43$ with or without a V5/His tag at the C-terminus. $\mathrm{Cx} 43$ was subsequently immunoprecipitated and the amount of ZO-1 that co-precipitated was assessed by Western blot. The results presented in Figure 3 show that the amount of ZO-1 that co-precipitates with $\mathrm{Cx} 43$ significantly decreases when a tag is added to the C-terminus of $\mathrm{Cx} 43$. The observation that a tag in the C-terminus of $\mathrm{Cx} 43$ reduces but not completely abolishes the interaction with ZO-1, can reflect either the residual expression of connexins with a PDZ binding motif in HEK293 cells or that a week interaction with ZO-1 still occurs in the absence of a PDZ binding domain in $\mathrm{Cx} 43$. To confirm that the levels of expression of both forms (tagged or untagged) of $\mathrm{Cx} 43$ were similar, the membranes were reprobed with antibodies to $\mathrm{Cx} 43$.

To further confirm that interaction of $\mathrm{Cx} 43$ with ZO-1 occurs through the PDZ2 domain of ZO-1, HEK293 cells were co-transfected with the untagged form of $\mathrm{Cx} 43$ and the PDZ2 domain of ZO-1. The PDZ2 domain of ZO-1 is the portion of the protein that interacts with the last 5 aminoacids of the C-terminus of $\mathrm{Cx} 43$. Thus, overexpression of the PDZ2 domain is likely to act as a competitive inhibitor for the binding of ZO- 1 to the C-terminus of $\mathrm{Cx} 43$. As shown in Figure 4A the amount of ZO-1 that coprecipitates with $\mathrm{Cx} 43$ is lower in cells that overexpress the PDZ2 domain as compared to controls. These results were further confirmed by immunofluorescence. Indeed, overexpres- 
A
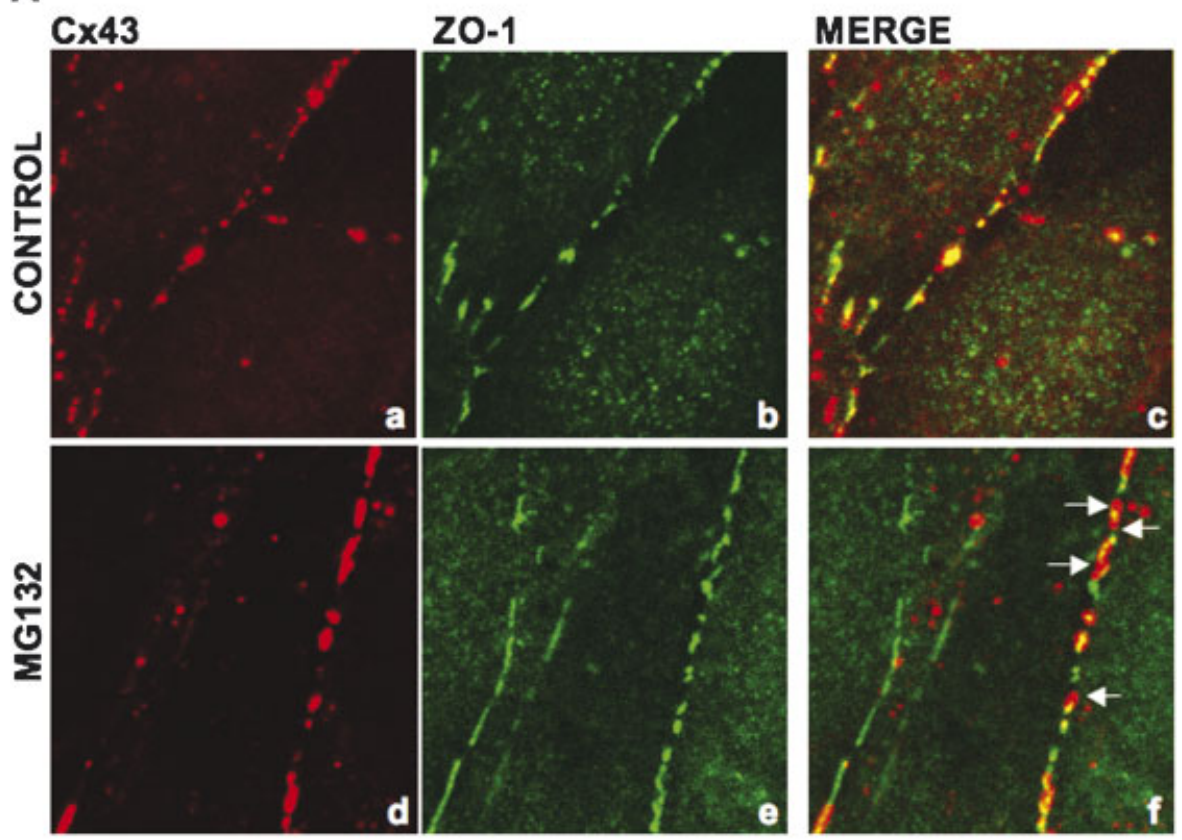

B

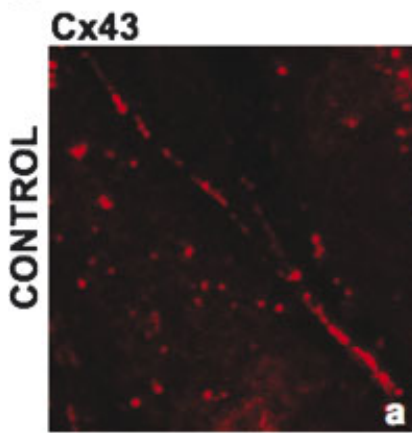

ZO-1

\section{MERGE}
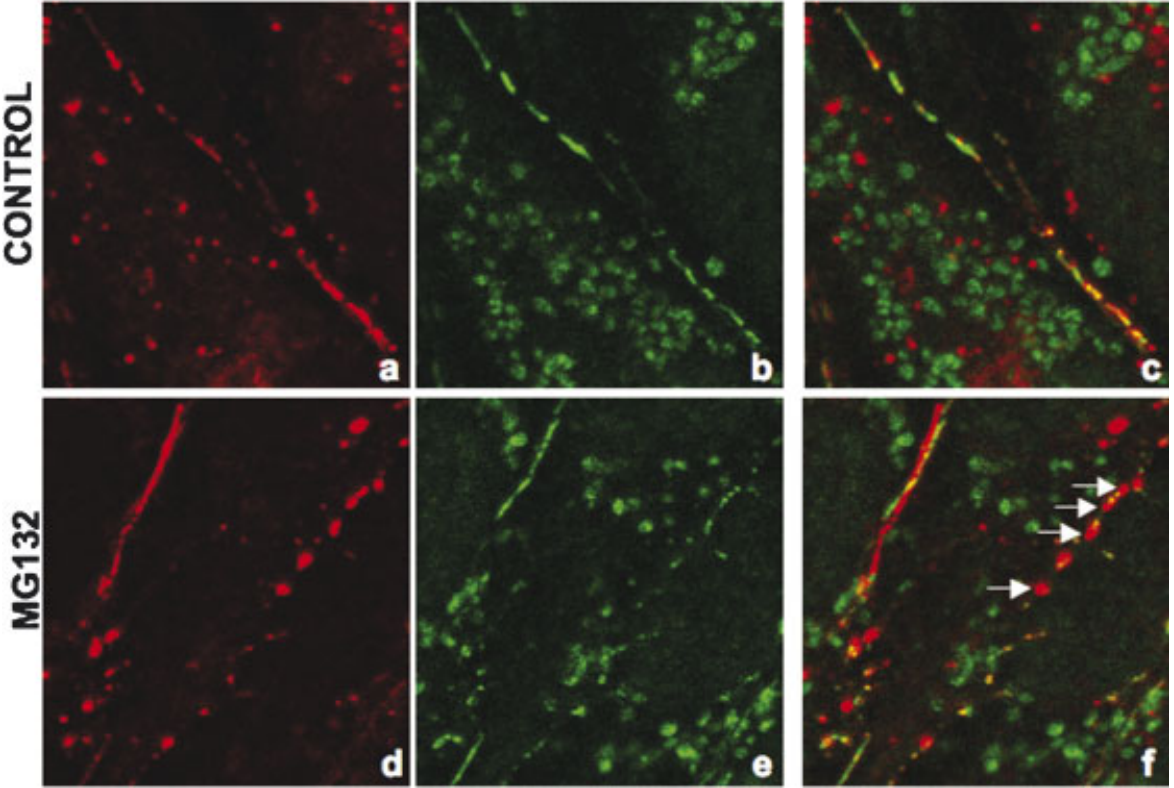

Fig. 2. Proteasome inhibitors reduce the colocalization of $\mathrm{Cx} 43$ with ZO-1. NRK cells were incubated with $20 \mu \mathrm{M} \mathrm{MG} 132$, for $2 \mathrm{~h}$, and then fixed either with PFA 4\% (A) or methanol/acetone (B) and double labeled with monoclonal antibodies directed against $\mathrm{Cx} 43$ (red) and polyclonal antibodies directed against ZO-1 (green) and imaged by confocal microscopy. Incubation with MG132 results in the accumulation of Cx43 at the plasma membrane and in a decrease in the extent of colocalization between Cx43 and ZO-1 (see arrows). [Color figure can be viewed in the online issue, which is available at www.interscience.wiley.com.]

sion of the PDZ2 domain results in a decreased co-localization between $\mathrm{Cx} 43$ and endogenous ZO-1 (Fig. 4B c, f; see arrows). These results show that overexpression of the PDZ2 domain of $\mathrm{ZO}-1$ disrupts the interaction between $\mathrm{Cx} 43$ and $\mathrm{ZO}-1$.

We do propose a model in which stabilization of $\mathrm{Cx} 43$, at the plasma membrane, depends on 


\section{Girao and Pereira}

IP: CX43

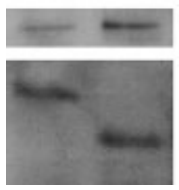

WB: $Z \mathrm{ZO}-1$

WB: $\mathrm{C} \times 43$

Tagged $\mathrm{C} \times 43$

Cx43

Fig. 3. The presence of a tag in the carboxyl terminal of Cx43 leads to a decreased interaction with ZO-1. HEK293 cells were transfected with $\mathrm{C} \times 43$ or V5-His tagged Cx43. Cx43 was immunoprecipitated using polyclonal antibodies against $\mathrm{C} \times 43$. Immunoprecipitated material was resolved by SDS-PAGE and immunoblotted with antibodies against ZO-1 and $\mathrm{Cx} 43$ as indicate in the picture.

the interaction of its C-terminus with the PDZ domain of ZO-1. We hypothesize that one mechanism by which the $\mathrm{Cx} 43 / \mathrm{ZO}-1$ interaction is regulated could be by degradation of an as yet unknown putative protein that binds to the C-terminus of $\mathrm{Cx} 43$. If this were the case, then binding of a tag to the C-terminus of $\mathrm{Cx} 43$ would, make the tagged $\mathrm{Cx} 43$ insensitive to the effects of proteasome inhibitors.

To verify this hypothesis, HEK293 cells were transfected either with a tagged or untagged $\mathrm{Cx} 43$. The transfected cells were then incubated in the presence or absence of the proteasome inhibitors for $3 \mathrm{~h}$, after which the cells were fixed and stained with antibodies directed against $\mathrm{Cx} 43$ and ZO-1. As shown in Figure 5A, when cells overexpressing the untagged form of $\mathrm{Cx} 43$, are incubated with MG132, a large punctate staining is revealed at

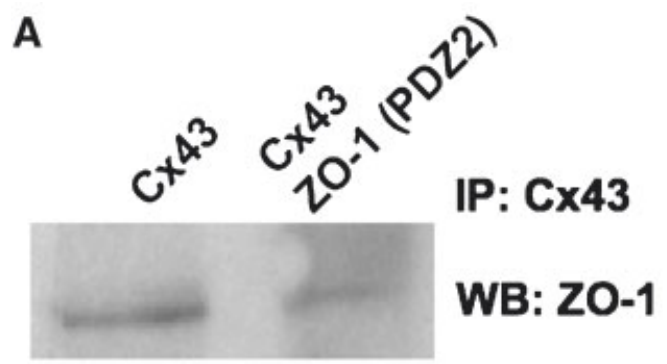

B

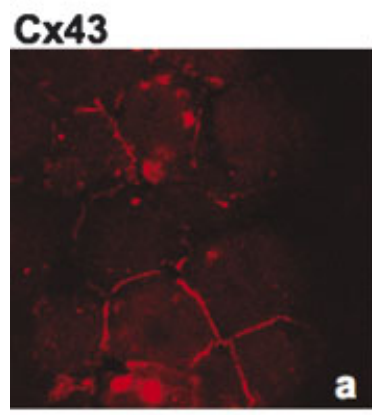

Zo-1
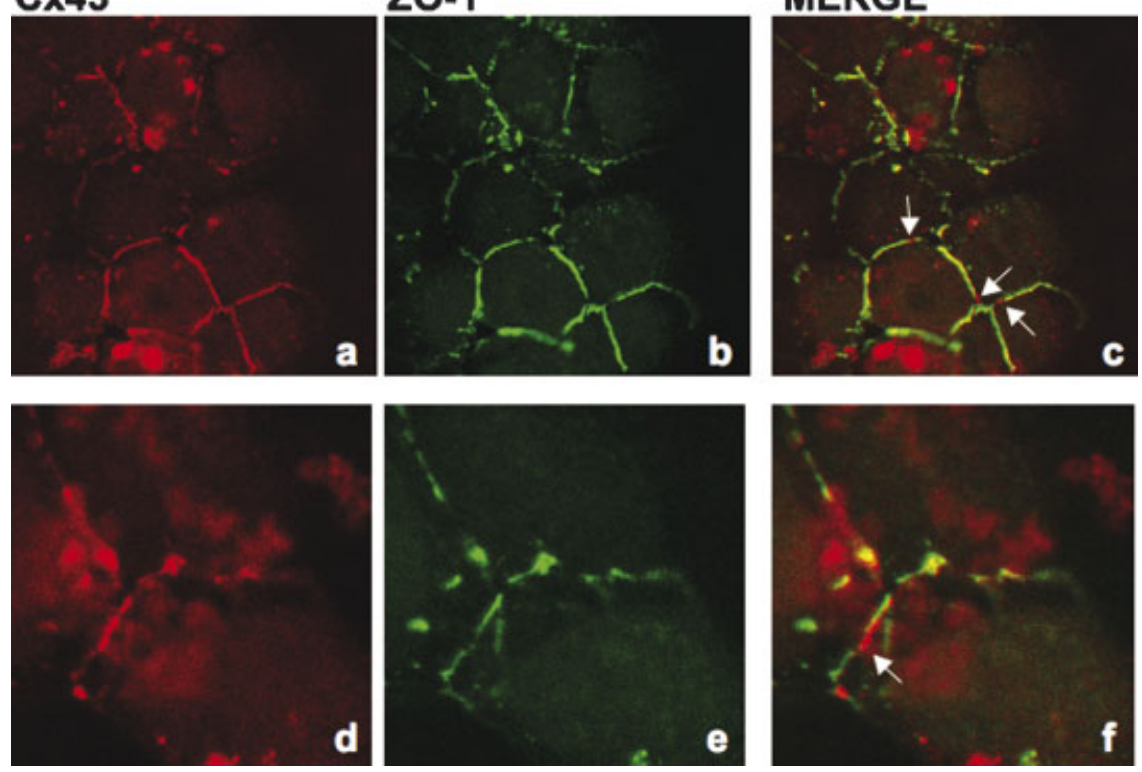

Fig. 4. Overexpression of the PDZ2 domain of ZO-1 disrupts interaction between Cx43 and ZO-1. HEK293 cells were transfected either with $\mathrm{C} \times 43$ alone or cotransfected withCx43 and the PDZ2 domain of ZO-1. Cx43 was immunoprecipitated using polyclonal antibodies against $\mathrm{C} \times 43$. Immunoprecipitated material was resolved by SDS-PAGE and immunoblotted with antibodies against ZO-1 (A). Alternatively, the cells were fixed and double labeled with monoclonal antibodies directed against Cx43 (red) and polyclonal antibodies directed against ZO-1 (green) and imaged by confocal microscopy (B). Overexpression of the PDZ2 domain of ZO-1 results in a decrease of colocalization of $\mathrm{Cx} 43$ and ZO-1 (arrows). [Color figure can be viewed in the online issue, which is available at www. interscience.wiley.com.] 
A
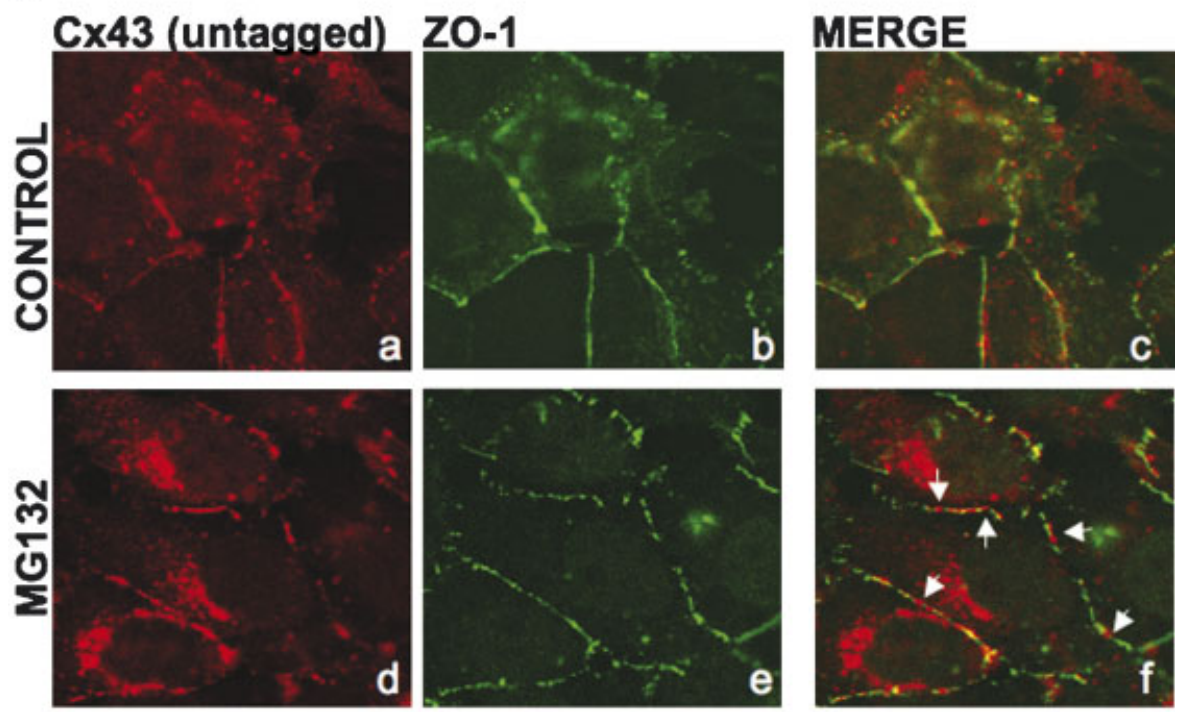

B
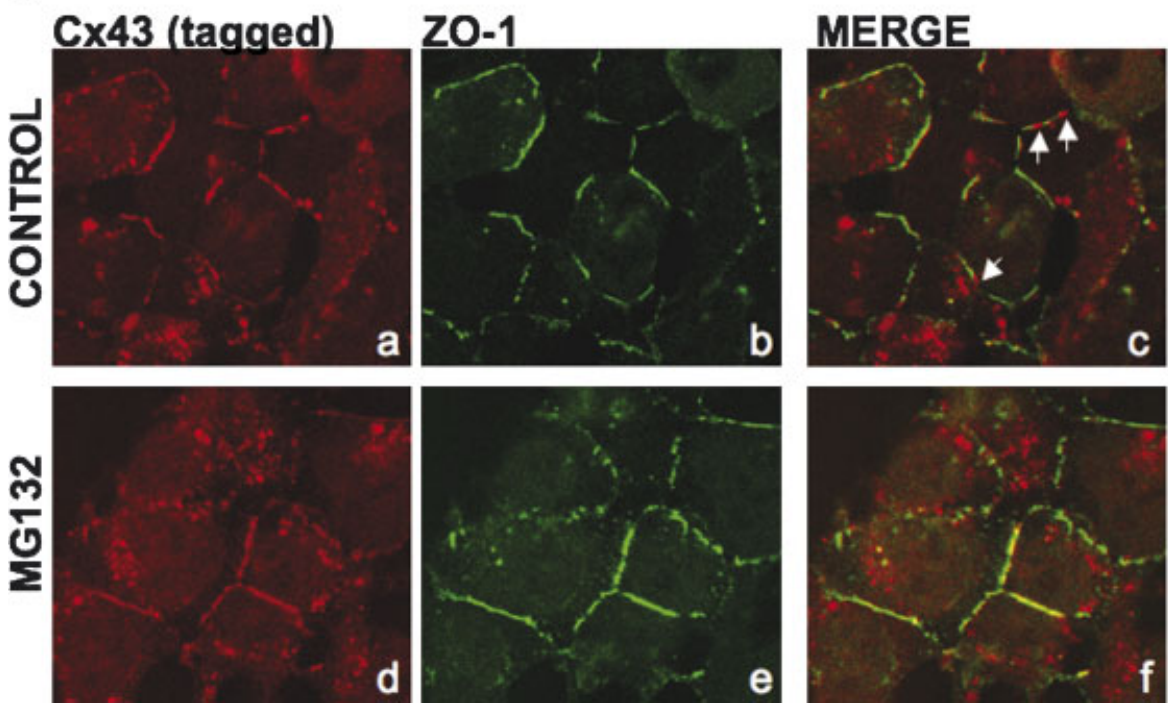

Fig. 5. The effect of proteasome inhibitors is abolished when $\mathrm{C} \times 43$ has a tag in the C-terminus. HEK293 cells transfected with untagged Cx43 (A) or V5-His tagged Cx43 (B) were incubated with MG132, for $3 \mathrm{~h}$. The cells were then fixed and double labeled with monoclonal antibodies directed against Cx43 (red) and polyclonal antibodies directed against ZO-1 (green) and imaged by confocal microscopy. Proteasome inhibition does not result in a decreased colocalization between $\mathrm{Cx} 43$ and ZO-1 when a tag is present in the C-terminus of $\mathrm{Cx} 43$. [Color figure can be viewed in the online issue, which is available at www.interscience.wiley.com.]

the plasma membrane (Fig. 5A d), as described before for distribution of endogenous $\mathrm{Cx} 43 \mathrm{NRK}$ cells. On the other hand, proteasome inhibition has no effect on cells that overexpress the tagged form of $\mathrm{Cx} 43$ (Fig. 5B d). Additionally, cells overexpressing the tagged form of $\mathrm{Cx} 43$ present larger punctate staining that do not colocalize with ZO-1 (Fig. 5B a-c, arrows), as compared to cells overexpressing the untagged form of $\mathrm{Cx} 43$ (Fig. 5A a-c).

\section{DISCUSSION}

The results presented in this study demonstrate that the proteasome regulates the interaction between $\mathrm{Cx} 43$ and $\mathrm{ZO}-1$, as revealed by coprecipitation experiments and immunofluorescence analysis. Indeed, incubation of the $\mathrm{Cx} 43$ expressing NRK cells with the proteasome inhibitor MG132 (or lactacystin), results both in a decrease in the amount of $\mathrm{ZO}-1$ that 
coprecipitates with $\mathrm{Cx} 43$ and in a diminished colocalization of the two proteins at the plasma membrane. The reduction of $\mathrm{Cx} 43-\mathrm{ZO}-1$ association is not accompanied by a reduction in the association between $\mathrm{Cx} 43$ and E-cadherin, suggesting that the proteasome is specifically affecting the interaction between $\mathrm{Cx} 43$ and ZO-1. Moreover, the presence of a tag in the C-terminus of $\mathrm{Cx} 43$, that prevents interaction with ZO-1, reverts the MG132-induced accumulation of large $\mathrm{Cx} 43$ plaques at the plasma membrane. Taken together, these findings suggest that interaction between $\mathrm{Cx} 43$ and $\mathrm{ZO}-1$ can be regulated by the proteasome. We and others have previously shown that the proteasome can regulate GJIC [Musil et al., 2000; Girao and Pereira, 2003; Fernandes et al., 2004]. Indeed, it is well established that proteasome inhibition leads to an accumulation of large plaques of GJ and, as a consequence, to an increase of GJIC. However, the mechanism whereby proteasome increases intercellular communication is still unknown. Recently, Hunter et al. [2005] proposed a model in which ZO- 1 controls the rate of $\mathrm{Cx} 43$ channel accretion at the periphery of GJ. The authors demonstrate that reduction of the interaction between $\mathrm{ZO}-1$ and $\mathrm{Cx} 43$ leads to the formation of abnormally large GJ plaques, with the concomitant accumulation of the phosphorylated forms of $\mathrm{Cx} 43$. This observation is consistent with the model proposed in the present study. For example, by preventing degradation of a putative $\mathrm{Cx} 43$-interacting protein, proteasome inhibitors could also prevent interaction between $\mathrm{Cx} 43$ and $\mathrm{ZO}-1$ resulting in the formation of large plaques of GJ. Moreover, we have shown before that phosphorylation acts as a signal targeting $\mathrm{Cx} 43$ for internalization by a proteasome-dependent mechanism [Girao and Pereira, 2003; Fernandes et al., 2004]. Indeed, it is conceivable phosphorylation plays an important role in proteasome-dependent regulation of the interaction between $\mathrm{ZO}-1$ and $\mathrm{Cx} 43$. Consistently, it was hypothesized that interaction between $\mathrm{Cx} 43$ and $\mathrm{ZO}-1$ is associated with increased internalization of $\mathrm{Cx} 43$ [Barker et al., 2002; Segretain et al., 2004] and that phosphorylation is a prerequisite for such $\mathrm{Cx} 43$ ZO-1 interaction and subsequent $\mathrm{Cx} 43$ endocytosis [Barker et al., 2002; Segretain et al., 2004]. These observations are consistent with the model proposed in this study for the proteasome-dependent internalization of $\mathrm{Cx} 43$.
That is, proteasome regulates association between $\mathrm{Cx} 43$ and ZO-1 and increased association between both proteins leads internalization of $\mathrm{Cx} 43$ and reduction of GJIC. According to this hypothesis, proteasome inhibition would result in the accumulation of $\mathrm{Cx} 43$ at the plasma membrane. This is likely to be relevant in conditions where the proteasome activity is inhibited or reduced, such as under oxidative stress. Indeed, it was previously shown that oxidative stress leads to the accumulation of $\mathrm{Cx} 43$ at the plasma membrane and, as a consequence, to a deregulation of GJIC [VanSlyke and Musil, 2002, 2005; Girao et al., 2004]. The results presented in this study envision an alternative mechanism that may associate deregulation of intercellular communication to oxidative stress. It is suggested that oxidative stress inhibits the proteasome, which, in turn leads to a poor interaction between $\mathrm{Cx} 43$ and ZO-1, thus enhancing GJ plaques formation and intercellular communication. In addition to its role in GJIC, connexins are also involved in cell communication with the extracellular environment via hemichannels. Cx43-based hemichannels are implicated in various processes in cell physiology and pathology including volume regulation [Quist et al., 2000], efflux of $\mathrm{NAD}^{+}$and ATP [Cotrina et al., 1998; Stout et al., 2002; Goodenough and Paul, 2003] and acceleration of cell death during metabolic inhibition [Contreras et al., 2002]. A previous study indicated that interaction of $\mathrm{Cx} 43$ with $\mathrm{ZO}-1$ could further contribute to regulate the pool of $\mathrm{Cx} 43$ that is involved in GJIC versus the pool that is involved in the non-junctional communication mediated by $\mathrm{Cx} 43$ hemichannels [Hunter et al., 2005]. Thus, it is conceivable that the changes in proteasome activity, may modulate, not only GJIC but also non-junctional communication through connexons, regulating other cellular processes such as apoptosis. Taken together, the results presented in this study suggest a new role for the proteasome in controlling GJIC, by regulating the association between $\mathrm{Cx} 43$ and $\mathrm{ZO}-1$.

\section{ACKNOWLEDGMENTS}

This study was supported by a Grant from the Portuguese Foundation for Science and Technology (FCT) (Programme POCTI). 


\section{REFERENCES}

Barker R, Price R, Gourdie R. 2002. Increased association of ZO-1 with connexin43 during remodeling of cardiac gap junctions. Circ Res 90:317-324.

Beardslee M, Lerner D, Tadros P, Laing J, Beyer E, Yamada K, Kleber A, Schuessler R, Saffitz J. 2000. Dephosphorylation and intracellular redistribution of ventricular connexin43 during electrical uncoupling induced by ischemia. Circ Res 87:656-662.

Bukauskas F, Jordan K, Bukauskiene A, Bennett M, Lampe P, Laird D, Verselis V. 2000. Clustering of connexin 43-enhanced green fluorescent protein gap junction channels and functional coupling in living cells. Proc Natl Acad Sci USA 97:2556-2561.

Contreras J, Sanchez H, Eugenin E, Speidel D, Theis M, Willecke K, Bukauskas F, Bennett M, Sáez J. 2002. Metabolic inhibition induces opening of unapposed connexin 43 gap junction hemichannels and reduces gap junctional communication in cortical astrocytes in culture. Proc Natl Acad Sci USA 99:495-500.

Cotrina M, Lin J, Alves-Rodrigues A, Liu S, Li J, AzmiGhadimi H, Kang J, Naus C, Nedergaard M. 1998. Proc Natl Acad Sci USA 95:15735-15740.

Duffy H, Ashton A, O’Donnell P, Coombs W, Taffet S, Delmar M, Spray D. 2004. Regulation of connexin43 protein complexes by intracellular acidification. Circ Res 94:215-222.

Fernandes R, Girao H, Pereira P. 2004. High glucose downregulates intercellular communication in retinal endothelial cells by enhancing degradation of connexin 43 by a proteasome-dependent mechanism. J Biol Chem 279: 27219-27224

Fujimoto K, Nagafuchi A, Tsukita S, Kuraoka A, Ohokuma A, Shibata Y. 1997. Dynamics of connexins, E-cadherin and alpha-catenin on cell membranes during gap junction formation. J Cell Sci 110:311-322.

Giepmans B, Moolenaar W. 1998. The gap junction protein connexin43 interacts with the second PDZ domain of the zona occludens-1 protein. Curr Biol 8:931-934.

Giepmans B, Verlaan I, Moolenaar W. 2001. Connexin-43 interactions with ZO-1 and alpha- and beta-tubulin. Cell Commun Adhes 8:219-223.

Girao H, Catarino S, Pereira P. 2004. 7-Ketocholesterol modulates intercellular communication through gapjunction in bovine lens epithelial cells. Cell Commun Signal 2:2.

Girao H, Pereira P. 2003. Phosphorylation of connexin 43 acts as a stimuli for proteasome-dependent degradation of the protein in lens epithelial cells. Mol Vis 9:24-30.

Gonzalez-Mariscal L, Betanzos A, Avila-Flores A. 2000. MAGUK proteins: Structure and role in the tight junction. Semin Cell Dev Biol 11: 315-324.

Goodenough D, Paul D. 2003. Beyond the gap: Functions of unpaired connexon channels. Nat Rev Mol Cell Biol 4: 285-294.

Harhaj N, Antonetti D. 2004. Regulation of tight junctions and loss of barrier function in pathophysiology. Int $\mathrm{J}$ Biochem Cell Biol 36: 1206-1237.

Hunter A, Barker R, Zhu C, Gourdie R. 2005. Zonula occludens-1 alters connexin43 gap junction size and organization by influencing channel accretion. Mol Biol Cell 16: 5686-5698.
Hunter A, Jourdan J, Gourdie R. 2003. Fusion of GFP to the carboxyl terminus of connexin43 increases gap junction size in HeLa cells. Cell Commun Adhes 10: 211-214.

Jin C, Martyn K, Kurata W, Warn-Cramer B, Lau A. 2004. Connexin43 PDZ2 binding domain mutants create functional gap junctions and exhibit altered phosphorylation. Cell Commun Adhes 11:67-87.

Jordan K, Solan J, Dominguez M, Sia M, Hand A, Lampe P, Laird D. 1999. Trafficking, assembly, and function of a connexin43-green fluorescent protein chimera in live mammalian cells. Mol Biol Cell 10:2033-2050.

Kumar N, Gilula N. 1996. The gap junction communication channel. Cell 84:381-388.

Laing J, Beyer E. 1995. The gap junction protein connexin43 is degraded via the ubiquitin proteasome pathway. J Biol Chem 270:26399-26403.

Laing J, Chou B, Steinberg T. 2005. ZO-1 alters the plasma membrane localization and function of $\mathrm{Cx} 43$ in osteoblastic cells. J Cell Sci 118:2167-2176.

Laing J, Tadros P, Westphale E, Beyer E. 1997. Degradation of connexin43 gap junctions involves both the proteasome and the lysosome. Exp Cell Res 236:482492.

Matemba S, Lie A, Ransjö M. 2006. Regulation of osteoclastogenesis by gap junction communication. J Cell Biochem 99:528-537.

Musil L, Le A, VanSlyke J, Roberts L. 2000. Regulation of connexin degradation as a mechanism to increase gap junction assembly and function. J Biol Chem 275:2520725215.

Quist A, Rhee S, Lin H, Lal R. 2000. Physiological role of gapjunctional hemichannels. Extracellular calcium-dependent isosmotic volume regulation. J Cell Biol 148:10631074.

Schubert A, Schubert W, Spray D, Lisanti M. 2002. Connexin family members target to lipid raft domains and interact with caveolin-1. Biochemistry 41:57545764.

Segretain D, Fiorini C, Decrouy X, Defamie N, Prat JR, Pointis G. 2004. A proposed role for ZO-1 in targeting connexin 43 gap junctions to the endocytic pathway. Biochimie 86:241-244.

Simon A, Goodenough D. 1998. Diverse functions of vertebrate gap junctions. Trends Cell 8:477-483.

Singh D, Lampe P. 2003. Identification of connexin-43 interacting proteins. Cell Commun Adhes 10:215220.

Sorgen P, Duffy H, Sahoo P, Coombs W, Delmar M, Spray D. 2004. Structural changes in the carboxyl terminus of the gap junction protein connexin43 indicates signaling between binding domains for c-Src and zonula occludens1. J Biol Chem 279:54695-54701.

Stout C, Costantin J, Naus C, Charles A. 2002. Intercellular calcium signaling in astrocytes via ATP release through connexin hemichannels. J Biol Chem 277:1048210488.

Toyofuku T, Akamatsu Y, Zhang H, Kuzuya T, Tada M, Hori M. 2001a. c-Src regulates the interaction between connexin-43 and ZO-1 in cardiac myocytes. J Biol Chem 276:1780-1788.

Toyofuku T, Yabuki M, Otsu K, Kuzuya T, Hori M, Tada M. 2001b. Direct association of the gap junction protein 
connexin-43 with ZO-1 in cardiac myocytes. J Biol Chem 273:12725-12731.

Toyofuku T, Yabuki M, Otsu K, Kuzuya T, Hori M, Tada M. 1998. Intercellular calcium signaling via gap junction in connexin-43-transfected cells. J Biol Chem 273:1519-1528.

VanSlyke J, Musil L. 2002. Dislocation and degradation from the ER are regulated by cytosolic stress. J Cell Biol 157:381-394.
VanSlyke J, Musil L. 2005. Cytosolic stress reduces degradation of connexin 43 internalized from the cell surface and enhances gap junction formation and function. Mol Biol Cell 16:5247-5257.

Wu J, Tsai R, Chung T. 2003. Role of catenins in the development of gap junctions in rat cardiomyocytes. J Cell Biochem 88: 823-835. 